\title{
Belgeo
}

Revue belge de géographie

1 | 2008

Aspects of the history of the Belgian geography and cartography

\section{Cartographie et géographie en Belgique à l'époque hollandaise : à propos des cartes de Monborne et de Wautier}

\section{Exemples régionaux}

Cartografie en geografie in België tijdens het Hollandse tijdperk: de Kaarten van Monborne en de Wautier

\section{Lisette Danckaert}

\section{(2) OpenEdition}

\section{Journals}

Édition électronique

URL : http://journals.openedition.org/belgeo/10197

DOI : 10.4000/belgeo.10197

ISSN : 2294-9135

Éditeur :

National Committee of Geography of Belgium, Société Royale Belge de Géographie

Édition imprimée

Date de publication : 31 mars 2008

Pagination : 87-104

ISSN : 1377-2368

Référence électronique

Lisette Danckaert, «Cartographie et géographie en Belgique à l'époque hollandaise : à propos des cartes de Monborne et de Wautier », Belgeo [En ligne], 1 | 2008, mis en ligne le 19 octobre 2013, consulté le 23 avril 2019. URL : http://journals.openedition.org/belgeo/10197 ; DOI : 10.4000/ belgeo.10197

Ce document a été généré automatiquement le 23 avril 2019

Belgeo est mis à disposition selon les termes de la licence Creative Commons Attribution 4.0 International. 


\title{
Cartographie et géographie en Belgique à l'époque hollandaise : à propos des cartes de Monborne et de Wautier
}

\author{
Exemples régionaux
}

Cartografie en geografie in België tijdens het Hollandse tijdperk: de Kaarten van Monborne en de Wautier

Lisette Danckaert

\section{Introduction}

1 La Revue bibliographique des Pays-Bas du 15 mai 1825, publication périodique de P.J. De Mat, commente la parution de la première livraison de l'Atlas universel de géographie... de Philippe Vandermaelen. En page 156, la présentation de l'ouvrage reprend le texte du prospectus de lancement qui indique des livres utilisés par le cartographe et cite : "grâce aux de Humboldt, aux Malte-Brun, aux Lapie, aux Mentelle, aux Guthrie, etc., cette science [la géographie] est devenue à la portée de tout le monde".

Très jeune déjà, Vandermaelen s'était attaché à parfaire ses connaissances géographiques et cartographiques. Son intérêt ne faiblit jamais. L'Etablissement géographique de Bruxelles, ouvert en mai 1830, se devait de tenir à la disposition de ses collaborateurs, des chercheurs et des étudiants curieux une riche documentation géographique, cartes et livres, comme en témoigne le catalogue de la vente des collections en $1880^{1}$, où 574 numéros concernent la géographie ancienne et moderne. Beaucoup de ces livres et périodiques étaient, certes, postérieurs à 1830, comme La Terre d'Elisée Reclus, mais les ouvrages plus anciens ne manquent pas, avec la Géographie mathématique, physique et politique de Mentelle et Malte-Brun, 1803, le Voyage en Sibérie fait en 1761 de Chappe d'Auteroche, publié en 1768, ou encore les Travels and discoveries in Northern and Central 
Africa de Clapperton en 1826, et même les nombreux tomes de la Description d'Egypte, relation de l'expédition française publiée de 1821 à 1829.

Cette curiosité pour les relations de voyage se retrouve dans le catalogue ${ }^{2}$ de la riche bibliothèque de Charles Van Hulthem ${ }^{3}$, avec un choix d'ouvrages couvrant le monde entier. Et ce n'était pas uniquement le côté spectaculaire de pays exotiques qui était mis en évidence par des livres illustrés ou des récits plus ou moins imagés. Sont également repris les 24 tomes en 12 volumes des Annales des voyages, de la géographie et de l'histoire de Malte-Brun, publiés de 1808 à 1815, ou les nombreuses éditions des voyages de James Cook.

\section{Géographie dans les collections de Van Hulthem}

4 A côté de cet engouement pour les découvertes, Van Hulthem collectionnait également des ouvrages de géographie de Malte-Brun (encore lui !), de Pinkerton avec sa Géographie moderne, une Géographie des enfans avec 21 cartes, un Manuel élémentaire pour la construction et le dessin de cartes géographiques de A.M. Perrot, Paris, 1830, et des Beginselen der landbeschrijvinge, livre édité à Bruxelles par P. de Bast en 1762. Cet ouvrage destiné à la jeunesse ne donne certes pas une haute idée des connaissances géographiques de son auteur. Il mentionne les deux hypothèses des cartographes contemporains concernant la Californie, île ou presqu'île...celle-ci rattachée à l'Asie ! Ce pays très fertile est fort peuplé dans le nord, mais les habitants n'ont pas de maisons; en été, ils se réfugient sous les arbres, en hiver, dans des cavernes.

Beaucoup plus intéressante est la seconde édition du Nouveau dictionnaire universel de la géographie moderne par F.D. Aynès, gros volume de plus de onze cents pages publié en 1818 à Paris. Il comporte des tableaux et des cartes du monde et de ses parties, ainsi que de l'empire français et des pays circumvoisins. Noms de lieux avec leurs coordonnées, les populations avec leurs manufactures et productions, les principaux sièges des villes se succèdent depuis "A, petite rivière de France, Loir-et-Cher" jusqu'à "Zyp (le), fameuse digue de la Nord-Hollande au travers de la presqu'île du Helder". Pour les pays, situation, limites, superficie, subdivisions sont complétées par de brèves descriptions des habitants et d'un grand choix de leurs productions, et pas seulement les plus importantes. Ainsi, pour la Perse, le tissage de tapis est une activité capitale, mais le pays reçoit des draps d'Angleterre et a de fréquents échanges commerciaux, par caravanes terrestres avec Alep et Bagdad, par mer dans le golfe Persique. Outre la description, un aperçu historique est donné pour les villes de l'Europe occidentale, surtout en rapport avec les opérations militaires françaises.

6 Les chaînes de montagnes ne sont pas oubliées, avec leurs différences de température et de végétation et leur rôle de réservoir d'eau. Malgré sa position, l'Atlas a des parties très froides et inhabitées. Certaines notions sont évidemment fausses, en rapport avec les connaissances insuffisantes de l'Afrique et des Etats-Unis, mais prouvent la prise en considération des renseignements récents, exacts ou non, rapportés par les explorateurs. Par exemple, croyance qui aura la vie dure, l'énorme chaîne censée traverser l'Afrique d'ouest en est, que Mungo Park s'imaginait avoir aperçue ${ }^{4}$, est citée, comme le fleuve Zaïre dans le Congo, qui toutefois se jette à l'est du continent dans la mer d'Ethiopie par une large embouchure. Les "Apalaches ou Allegany" sont de très hautes montagnes qui semblent être la ligne de partage des eaux du Pacifique et de l'Atlantique - les Rocheuses étant inconnues, comme le centre. Il ne faut pas oublier que lors de la vente de la 
Louisiane, les Français ignoraient quel territoire réel ils cédaient. Tout erronées que puissent être ces rubriques du Dictionnaire d'Aynès, elles témoignent de grands progrès dans le regard porté sur la géographie par rapport aux ouvrages antérieurs, qui se contentaient parfois d'une énumération fastidieuse de superficies et distances à apprendre par coeur.

Bien entendu, Van Hulthem n'est pas vraiment représentatif de l'ensemble de la population, mais pour ce passionné de botanique et de bibliophilie, avoir tant d'ouvrages concernant les sciences de la terre, s'intéresser à l'amélioration de l'agriculture et des bâtiments ruraux et instruments agricoles, au creusement d'un canal entre Escaut et Rhin ${ }^{5}$, voilà qui témoigne d'une ouverture vers une évolution de la géographie humaine ou politique, suivant la terminologie de l'époque.

Cependant, Charles Van Hulthem ne semble pas avoir acquis un livre disponible en Belgique, la Géographie du Royaume des Pays-Bas, éditée à Bruxelles par Stapleaux en 1819. L'auteur, L. Dewez, estime que dans les ouvrages de géographie universelle il n'y a presque rien sur la Belgique et que donc les jeunes gens ne connaissent pas leur pays. Il dit avoir réalisé un travail original, qui n'est copié sur aucun autre. Après la situation générale et les limites, les trois fleuves principaux (Rhin, Meuse, Escaut) avec leurs affluents et quelques villes riveraines, sont étudiées les dix-huit provinces en deux groupes, méridionales et septentrionales. L'ouvrage pédagogique est, en fait, un mélange de brefs rappels historiques, de notions administratives et de géographie. Rivières, nature des sols avec leurs qualités et les productions, tant minérales que culturales (labours sur les bonnes terres, prairies dans les zones humides, bois, landes), procédés d'exploitation avec insistance sur l'amélioration apportée par le travail humain sur des sols ingrats, parfois les assolements, sont complétés par le "caractère" des habitants. Ces portraits stéréotypés étaient traditionnels dès la fin du XVIIIe siècle et se perpétueront encore longtemps.

9 A cette époque, plusieurs auteurs soulignent l'importance de la consultation de globes et de cartes pour connaître la Terre, et ceci même pour les enfants comme le désire P.N. De Muyt en 1824 dans son Aardrijksbeschrijving voor kinderen. Les documents cartographiques d'origines diverses, principalement allemandes et françaises, ne manquaient pas, et étaient vivement recommandés comme indispensables à l'étude par A.F. Busching dans son Introduction à la géographie... des Etats de l'Europe, parue en traduction à Bruxelles chez Lemaire en 1786. Cartes générales et régionales dressées par des militaires ou publiées par des civils côtoyaient les plans de villes tant manuscrits que gravés.

Outre des atlas anciens terrestres et maritimes et des recueils factices, ainsi qu'un bel exemplaire de la carte marchande de Ferraris et un de l'atlas de Fricx, Van Hulthem possédait des cartes enroulées et neuf portefeuilles contenant probablement des cartes volantes. Malheureusement, le catalogue ne les énumère pas et il n'est donc pas possible de les répertorier. Elles auront vraisemblablement été intercalées de nombreuses années plus tard dans les collections de la Bibliothèque royale de Belgique suivant le système de classement usuel, ce qui exclut actuellement une identification certaine de la provenance. Cependant, aucune carte du prolifique Jean-Baptiste De Bouge, cartographe longuement étudié par Claire Lemoine-Isabeau ${ }^{6}$, n'est citée dans le catalogue sous le nom de cet auteur ni sous le titre, et il semblerait donc que Van Hulthem ait ignoré cette importante production contemporaine. 


\section{Une série peu connue : la carte de Monborne}

11 Une autre oeuvre qui aurait dû logiquement intéresser un bibliophile aussi averti, celle de Madame Monborne ${ }^{7}$, manque également. Elle a pourtant été annoncée dans la Revue bibliographique en 1824 comme devant être la "Carte topographique, physique et militaire en soixante feuilles, de la limite des royaumes de France et des Pays-Bas, à laquelle on a joint les environs de Bruxelles". La série de cartes est censée améliorer la connaissance du relief, de l'habitat, etc., donc de la géographie, mais n'a jamais été terminée par cette dame. Plusieurs feuilles sont signées. L'échelle est $1 / 16100$. En recherchant dans plusieurs bibliothèques et dépôts d'archives, C. Lemoine-Isabeau a retrouvé 13 (14 ?) feuilles pour la zone frontalière, qui ne sont pas toutes présentes à la Bibliothèque Royale. De même, 15 feuilles sur les 20 probables de la carte des environs de Bruxelles sont conservées dans cette institution, une seizième ayant été localisée ailleurs. Sont-ce les seules qui ont été lithographiées sur le total prévu, ceci par manque d'intérêt soutenu des acheteurs potentiels, bien qu'il y ait eu des souscripteurs importants, ou est-ce la source de renseignements de Madame Monborne qui s'est tarie ? C. Lemoine avance prudemment l'hypothèse $^{8}$ que l'ingénieur géographe $\mathrm{E}$. Breugnot (ou Breugnot-Monborne), participant aux travaux de cartographie de la frontière franco-belge, aurait pu transmettre des données à une parente pour édition privée. Mais les cartes à 1/25000 des Militaire Verkenningen ou Reconnaissances militaires, dont quelques feuilles présentent une certaine similitude avec la petite zone du territoire frontalier reproduite par Madame Monborne, n'étaient probablement pas destinées à être répandues telles quelles dans la société civile. Vaste entreprise cartographique militaire pour représenter la partie méridionale du royaume des Pays-Bas, elle fut interrompue par la révolution de 1830. Les cartes manuscrites ont été étudiées par Lieve Hens-Vercauteren et, plus récemment, par Freddy Janssens ${ }^{9}$.

12 Basées sur des travaux de triangulation, des sources diverses telles les réductions des plans cadastraux existant et des relevés minutieux sur le terrain, les cartes des Militaire Verkenningen (dorénavant M.V.) représentent le relief avec des hachures. Ce procédé se retrouve, un peu simplifié, chez Monborne et, lorsque la comparaison est possible, la ressemblance est troublante. Le fac-similé de deux feuilles des M.V. a été confronté aux trois cartes lithographiées correspondantes. Les orthographes respectives sont conservées.

13 La feuille I.3 de Monborne montre la région de La Panne et Coxide (figure 1), reprise sur une petite partie de la feuille 12, Nieuwpoort, des M.V., avec Caxyde. Là où Monborne indique un massif de dunes avec den hoogen Blecker, les M.V. amorcent seulement le groupe de dunes, d'un dessin différent. Par contre, les formes des parcelles de prairies individualisées sont semblables, les terres arables restant en blanc. Les couleurs diffèrent, les jardins étant en jaune avec des arbres éventuels, les bâtiments noirs et des bois le long des dunes et route côtière sur la carte imprimée ; pas de jardins ni bois et des maisons rouges sur les M.V. Il y a plus de bâtiments dans les dunes traversées par des chemins, comme le chemin de Dunkerque et les noms des fermes et les toponymes sont plus nombreux chez Monborne: Groete quente Ferme, Klein quente, Belle Videlle, etc. Paelement Grachgt et Pavé de Furnes restent également anonymes sur les M.V., Zoet Inval Cabaret manque et la ferme St Laurens Hof n'a que trois bâtiments contre cinq; le tracé des routes est identique. Au sud-ouest de Coxide, le canal de l'Angilis correspond à 
l'Oost Beek et, à l'est, un petit tronçon anonyme de canal fait partie du kanaal de Langhelege Gracht qui rejoint le kanaal van Veurne sur les M.V.

Figure 1. Monborne f.I.3, La Panne.

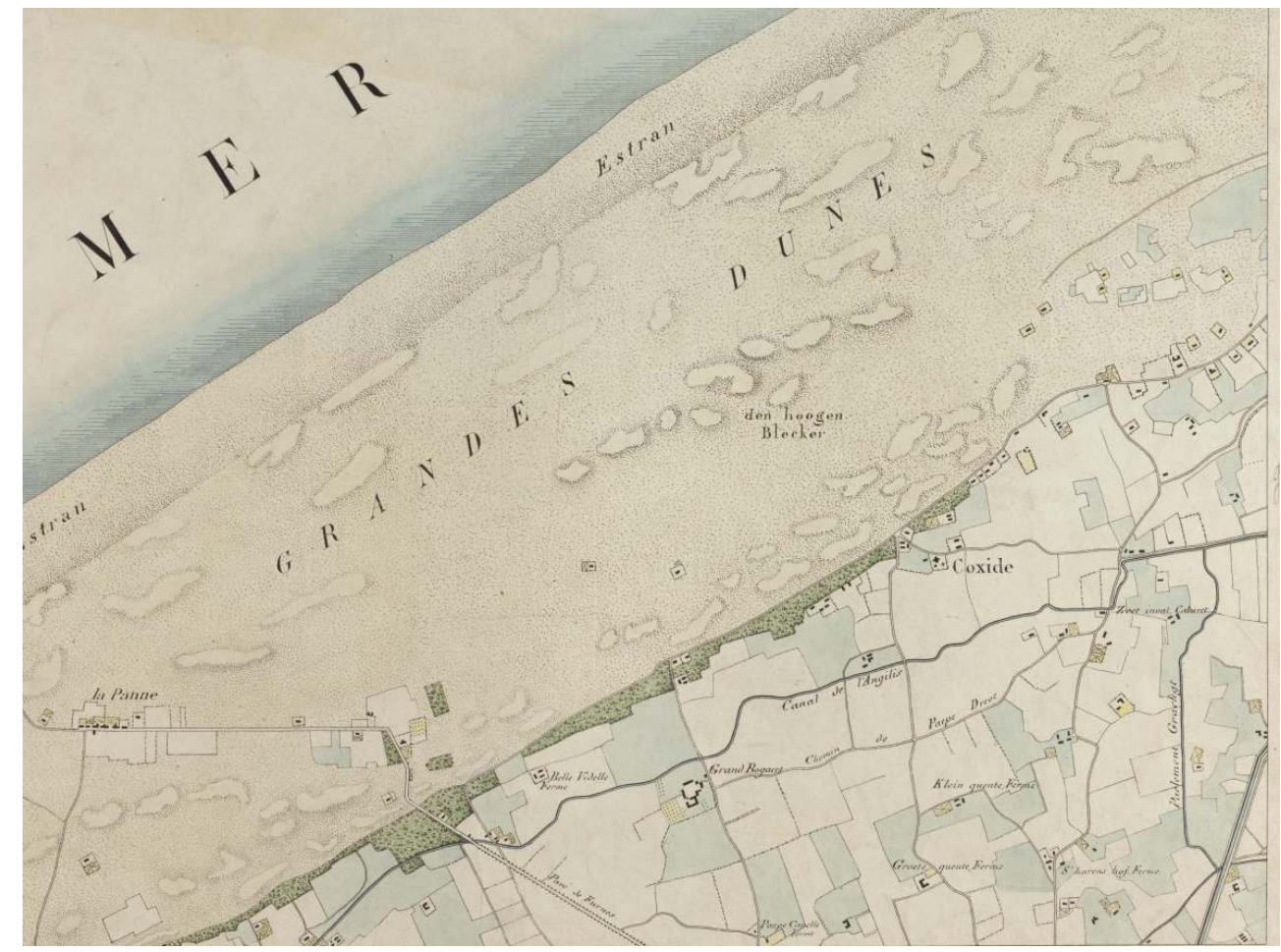

Les feuilles Q.7 et Q.8, Menin-Wervick, de Monborne (figure 2) donnent les distances à la méridienne et à la perpendiculaire à la méridienne de Paris, comme le faisait la carte marchande de Ferraris. Ces deux feuilles correspondent à la feuille 29 Meenen-WervickComines $^{10}$, signée par le colonel Van Gorkum, directeur des M.V. Le cours de la Lys et le réseau de fossés sont presque semblables, à l'exception d'un tout petit bras au sud-ouest de Wervick et de trois minuscules pointes qui manquent sur les M.V., où une boucle de la rivière est un peu plus serrée au nord de Bousbecque. Toutefois, la carte manuscrite mentionne près de Meenen une Groote Inondatie au sud-ouest et une Kleine à l'est. La ville même est représentée par ses îlots construits, en rouge, avec mise en évidence dans le nord des quatre casernes et du couvent des capucins ; au sud, l'église principale et, au centre, l'hôtel de ville. Toutes ces constructions, en noir, sont individualisées chez Monborne et il y a une rue supplémentaire, ainsi que des jardins. Le tracé des fortifications est pareil. Les routes et l'emplacement des bâtiments dans les environs sont les mêmes sur les deux documents, sauf des moulins qui manquent sur la lithographie. 
Figure 2. Monborne f.Q.7, Menin.

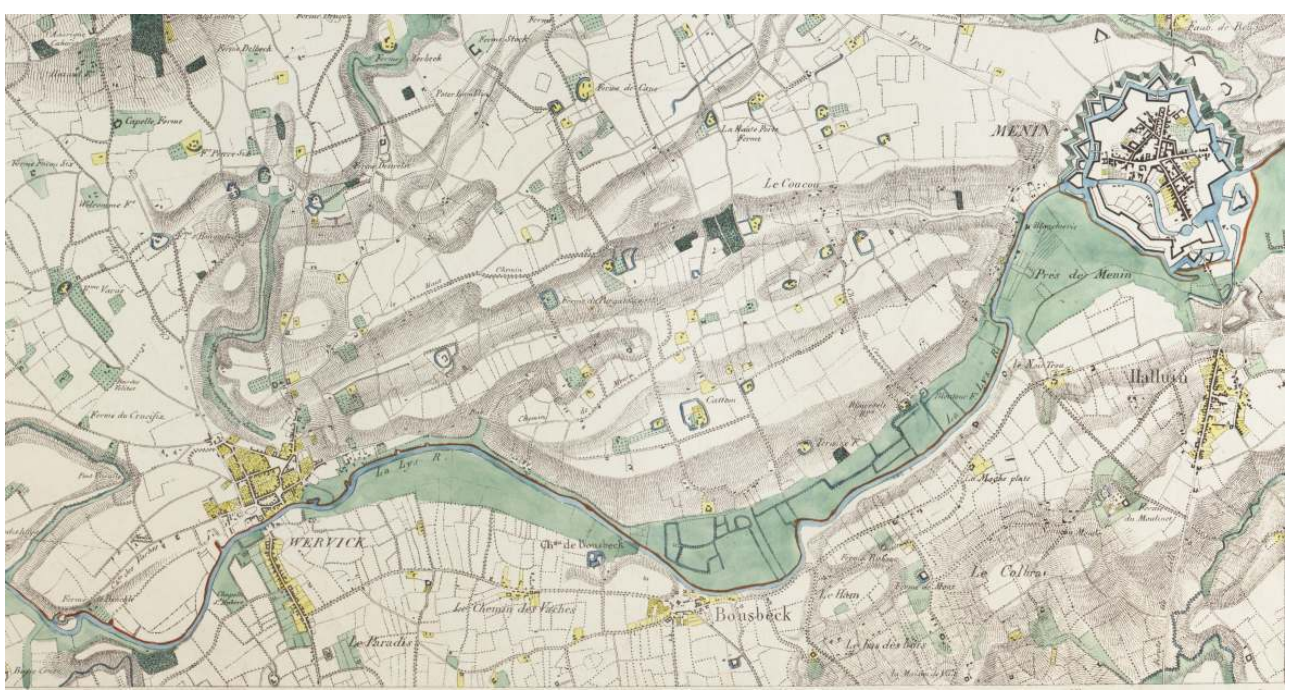

En résumé, les ressemblances sont suffisantes pour qu'il ne paraisse pas impossible que, parmi d'autres sources, Madame Monborne ait eu connaissance -indirectement de renseignements généralement tenus plus secrets. La Revue bibliographique de 1824 mentionne, en page 196, dans sa présentation des soixante feuilles, que plusieurs administrations, dont le Dépôt général des Fortifications, et les ministres de l'Intérieur de France et de Belgique [sic] ont souscrit à la collection. Y aurait-il eu une espèce de symbiose entre l'armée et le privé pour un partage des frais d'édition d'une entreprise assez considérable, restée inachevée ? Peut-être y a-t-il dans les archives néerlandaises des documents qui pourraient apporter un début de réponse à cette énigme.

Pour la carte des environs de Bruxelles, le problème est différent, puisque les M.V. ne couvraient pas encore cette partie du territoire. Les plans de la ville proprement dite étaient déjà nombreux ; les faubourgs étaient figurés sur le Plan topographique... dressé par De Bouge, publié en 1816 par Maillart à l'échelle 1/7100 et repris dans l'Itinéraire de Bruxelles et de ses faubourgs ou Guide des étrangers. Plus largement et à échelle plus petite, les environs sont repris sur la Carte topographique de Bruxelles et de ses environs, réalisée en 1810 par Guillaume de Wautier ${ }^{11}$, qui publia également des cartes des grands-routes pour compléter le plan de la ville. Ces itinéraires indiquent les principales constructions le long des chaussées -surtout auberges et cabarets!-, mais aussi les grands arbres et les événements marquants. Ainsi, bien que les limites traversées par les routes soient celles des départements, les faits d'armes de 1815 ont été gravés, introduisant une ambiguïté de datation. La bande représentée étant étroite, ces itinéraires n'ont pas pu apporter beaucoup de renseignements aux lithographes François Williaume Frères qui travaillaient sous la direction de Madame Monborne et l'échelle des cartes générales était bien petite pour un usage commode de report à 1/16100.

\section{Bruxelles chez Monborne}

La carte des environs de Bruxelles de Madame Monborne comprend, outre la ville en grisé, les faubourgs où le peuplement prédomine dans le centre et le long des routes et où la superficie des "jardins", c'est-à-dire principalement des cultures maraîchères, est importante. Des communes assez proches, comme les Woluwé et Watermael, restent 
rurales et la carte s'étend jusqu'à des villages plus lointains : Neeryssche, Cortenberg, Tourinnes, Beauvechain, et l'extrême sud-est de la feuille 4 frôle Louvain. La présentation est la même que celle des cartes de la frontière, avec couleurs identiques et relief hachuré; les étangs asséchés sont différenciés des nombreux plans d'eau qui existent un peu partout (figure 3), même aux abords de la ville, par exemple à Etterbeek, Molenbeek et Forest.

Figure 3. Monborne f.6, Bruxelles/Anderlecht.

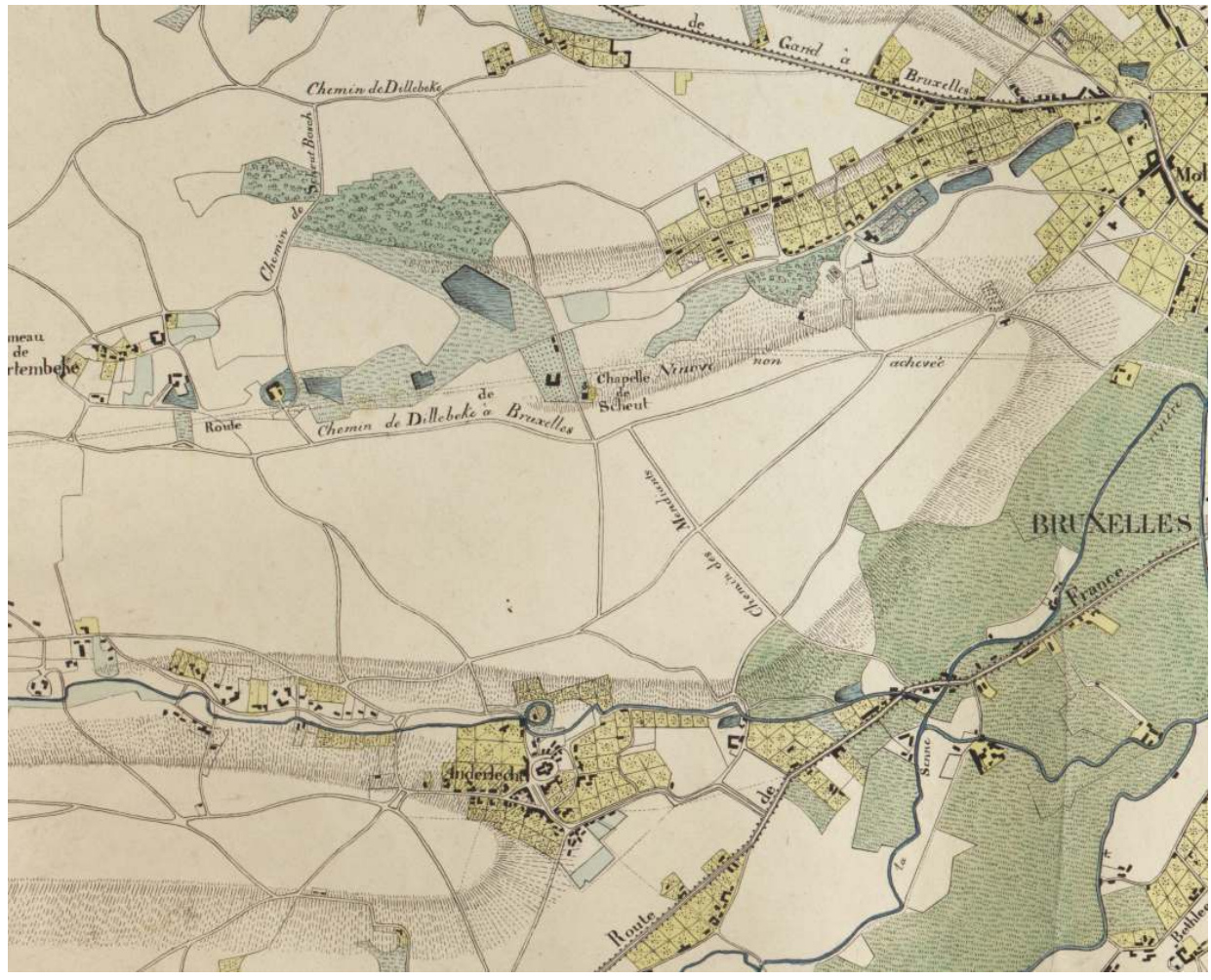

Routes et cours d'eau portent leur nom, ainsi que quelques chemins, tel le chemin du Trou des Moutons près d'Auderghem. Les toponymes sont assez nombreux: Bois de Fondroi et de Berthem, couvent de Terbanck et dans la forêt de Molendael, sur la feuille d'Opvelp, traversée par la chaussée de Louvain à Namur, la ci-devant abbaye de Valduc. Parmi les cabarets est mentionné "le ci-devant dernier sou" !

Les diverses feuilles ont été déposées par Aug. Desfossés ou R. Brochard et plusieurs portent dans la marge la signature à la plume de Madame Monborne.

\section{Comparaison Wautier-Monborne}

Comme il existe à la Bibliothèque Royale une carte manuscrite presque contemporaine des environs de Bruxelles, il était tentant de faire une rapide comparaison des deux documents, même si les auteurs respectifs n'ont vraisemblablement pas eu connaissance du travail de l'autre. Le territoire représenté sur les feuilles dessinées par Guillaume de Wautier de Beren n'est pas exactement le même, car elles s'étendent vers le sud au lieu de l'est. Au fur et à mesure de l'éloignement de la cité, le dessin est inachevé ${ }^{12}$. Les feuilles terminées, tracées à la plume à l'encre noire et rouge et coloriées, sont dressées à l'échelle 1/6100. Riches en détails, elles montrent les dénivellations par des hachures, y 
compris pour les chemins creux et le talus des fortifications. Les terres arables sont rendues selon la convention militaire du XVIIIe siècle (traits parallèles au sein de figures plus ou moins géométriques, mais ne délimitant pas les parcelles, sauf celles qui sont bordées par un chemin ou des haies). Les prairies marécageuses ou non, les bois, les cultures maraîchères et jardins, les étangs sont différenciés. Les noms des propriétaires des grandes maisons de campagne et des fabriques autour de Bruxelles sont souvent repris, avec pour les établissements industriels mention du genre de production : coton, papier, poudre à canon, etc. Les moulins à vent et surtout à eau, utilisés pour la production d'huile ou de farine, sont nombreux; des tours montrent l'importance du télégraphe Chappe dans le paysage. Un toponyme savoureux, tel l'Endroit aux Fromages appelés Hette Kees à Droogenbosch, est peut-être une allusion à la fabrication locale de cette spécialité bruxelloise.

21 Les îlots bâtis de Bruxelles sont en rouge, avec mise en évidence des grands édifices, soit par une teinte plus foncée, soit par le profil des églises; le parc et quelques jardins sont dessinés, les prairies et les blanchisseries dans l'ouest sont encore étendues. Les restes des fortifications ne sont pas déblayés et la Grosse Tour est toujours dessinée malgré la mention "démolie en 1808". Parmi les noms des maisons se remarque celui du célèbre carrossier Simons dans le nord et la demeure de l'auteur, qui localise également sa maison de campagne.

Les limites des huit sections sont tracées, mais le redressement du boulevard au nord n'est pas commencé. Une bande qui a été collée sur la feuille montre la terrasse de la future chaussée de Ninove, levée par Wautier en 1821 et 1822 ; il y a également un papillon avec des rues, déjà ouvertes à la fin du siècle dans l'ouest. L'état de la ville correspond environ à la situation vers 1819 , ce qui est contredit par d'autres feuilles.

Sur la carte lithographiée de Monborne, le boulevard de ceinture est aménagé avec l'accroissement de territoire dû au redressement, avec un petit bassin supplémentaire dans l'angle septentrional et, vers l'est, un triangle où sera érigé l'observatoire. La forme actuelle du pentagone est ainsi amorcée. L'aspect général est donc plus moderne. La place des Barricades est également tracée. La rue Royale atteint le boulevard, alors qu'elle s'arrête à la place de Louvain chez Wautier, qui dessine un parc plus trapu et représente plus d'impasses et des escaliers à l'intérieur de la ville. Il reste de l'eau dans les fossés occidentaux et même passé la porte de Hal chez Monborne; les vestiges des remparts sont laissés en blanc comme les terres arables, alors que les prairies marécageuses de la vallée sont pareilles sur les deux cartes (figures 3 et 4). Aux alentours immédiats, le cours de la Senne est assez semblable, un peu plus sineux chez Wautier, avec cependant ici un petit bras supplémentaire formant un îlot où est établie une manufacture d'indienne. Entre le hameau d'Aa et Papsem à Anderlecht, il y a une cascade remarquable dans un petit méandre recoupé où est établi un moulin (figure 5). Le long de la rive droite de la rivière, là où Monborne laisse un blanc, il $\mathrm{y}$ a des Franches Garennes. Cette indication se retrouve fréquemment sur les diverses feuilles de la carte manuscrite, souvent comme "ci-devant". 
Figure 4. Wautier f.4 planchette 1/3 Anderlecht.

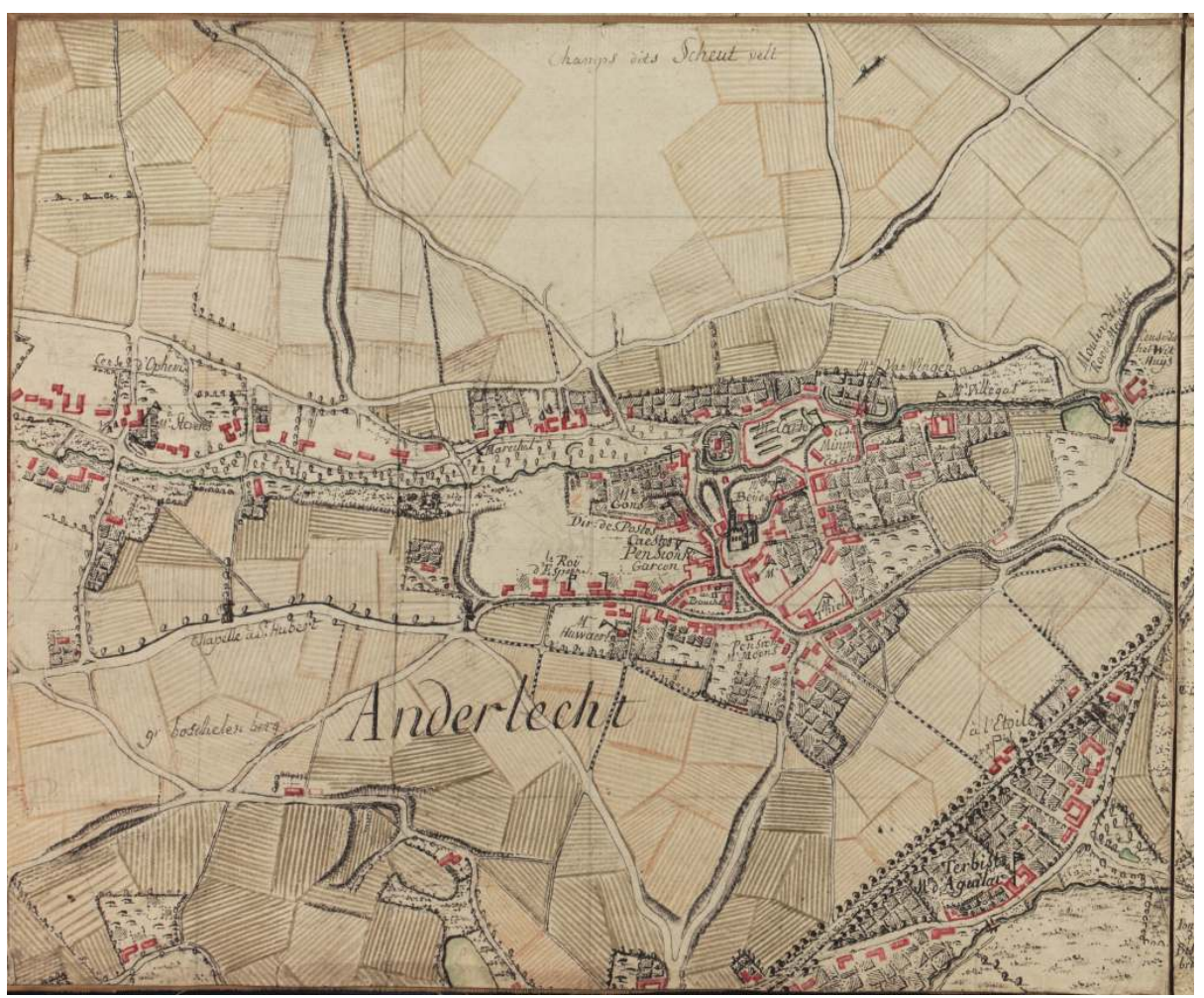

Figure 5. Wautier f.4 planchette $3 / 1$ Aa et cascade.

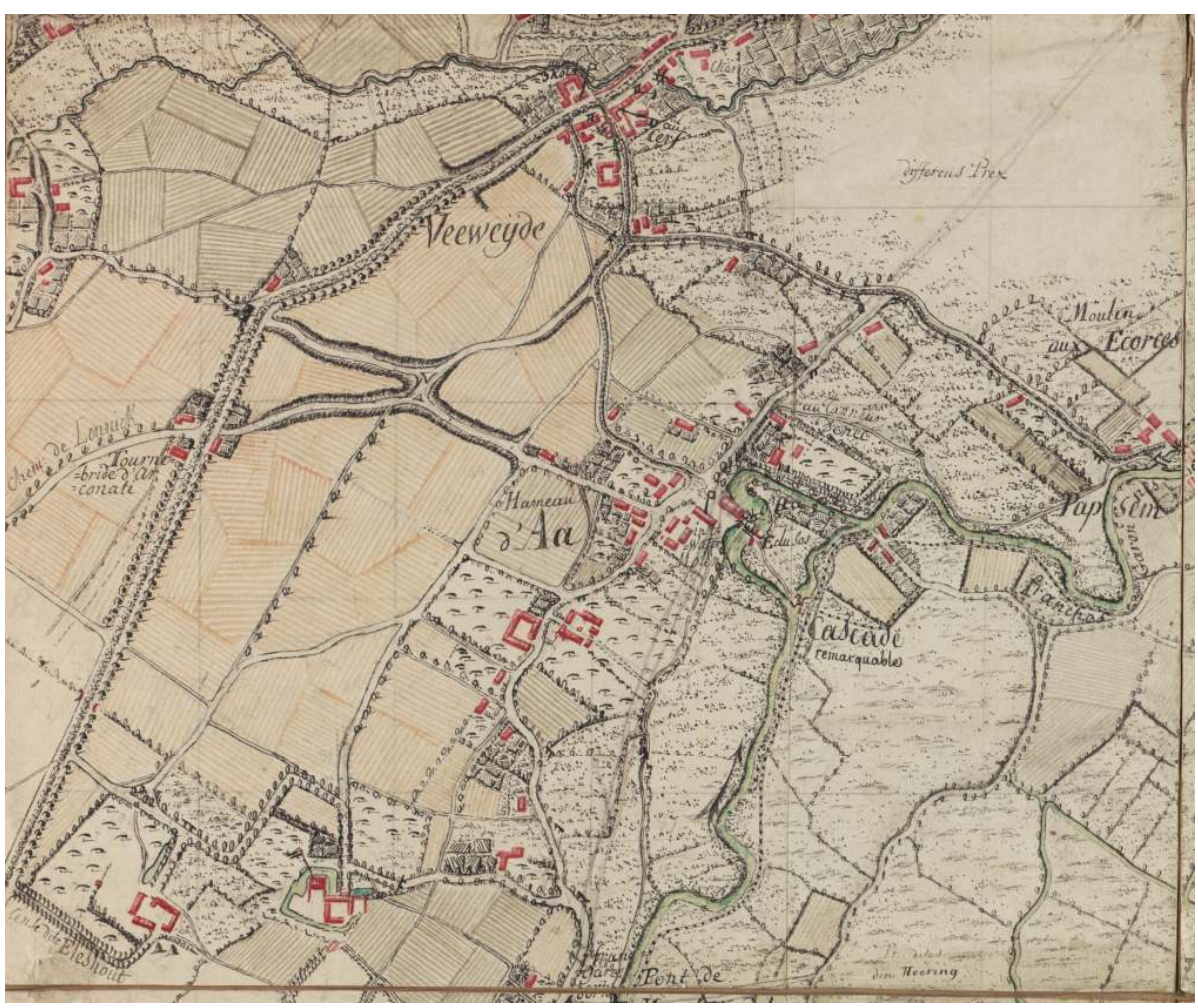

Comme dit plus haut, Wautier a ajouté le tracé futur de la chaussée de Ninove; celle-ci est dessinée en pointillé, comme non achevée chez madame Monborne, qui nomme la 
chaussée de Mons route de France et montre une chaussée de Flandre moins coudée. Elle omet également le nom du faubourg Martin à Molebeke, Moolenbeek sur le manuscrit. Par contre, les chemins près de la chaussée de Dillebeke à Bruxelles diffèrent du tracé anonyme chez Wautier, où le bois de Scheut est traversé par un chemin qui longe seulement ce bois sur la lithographie. Lieux-dits et noms des établissement, tant en ville que dans les environs, sont beaucoup plus nombreux chez Wautier qui reprend même les pensionnats de garçons des faubourgs.

\section{Caractéristiques de la carte de Wautier}

Aspect général différent mais contenu finalement assez comparable quant au tracé des ruisseaux, chemins, constructions et espaces cultivés. Rien ne permet cependant de croire à une parenté quelconque. Les deux auteurs ont peut-être pu utiliser des documents cadastraux comme base partielle, outre des travaux personnels. Guillaume de Wautier avait été formé à l'Artillerie et à l'Ecole d'Hydraulique. Il a certainement fait fonction ensuite de géomètre-arpenteur, puisqu'il atteste lui-même avoir levé la future chaussée de Ninove en 1821-22. Et ici apparaissent implicitement des renseignements quant à la façon de dresser des cartes. Les feuilles inachevées fourmillent de données chiffrées, difficiles à interpréter, qui concernent des distances et des angles. Les distances sont indiquées le long des routes, qui sont les premiers éléments tracés à l'encre dans les carrés préparés ${ }^{13}$, mais aussi le long de chemins encore crayonnés ou même en direction d'un croisement, d'un arbre remarquable ou d'un clocher. D'après Jan De Graeve, géomètre-expert qui a aimablement fourni ces renseignements, les visées d'angle ont dû être faites avec un appareil de mesure horizontal divisé en 400 grades. Ce spécialiste a, en effet, effectué un contrôle des nombreuses lignes rayonnant du centre de Braine-l'Alleud. Les opérations originales ont été faites à contresens des aiguilles d'une montre à partir d'un point de départ qui ne semble pas être le nord, bien que l'ensemble des cartes soit orienté ainsi. Beaucoup de ces visées sont évidentes, faites entre clochers, centres de villages ou vers un arbre élevé situé à un carrefour de chemins dans la campagne. Mais assez nombreuses sont celles qui paraissent se perdre dans la nature, ce qui ne facilite pas la compréhension du procédé. Il y a par exemple au nord-est de Nivelles un "ce Raion est bon", qui ne passe pourtant pas par le prieuré voisin.

Pour les distances indiquées le long des routes, l'unité employée (pas, aune, mètre, toise ? ) a dû être recherchée par rapport à l'échelle connue et confrontée à une carte récente. Elle vaut (presque) deux mètres, donc un peu plus grande que la toise française de l'ancien régime, qui donnerait une échelle ne correspondant pas au 1/6100 mesuré. Au dos de la première feuille, une échelle de 1000 aunes ou mètres est tracée au crayon et confirme l'usage du système métrique promu par le roi Guillaume Ier, qui tolérait cependant les anciens noms.

Les pentes, parfois qualifiées de descente difficile, et les encaissements des routes sont indiqués, des hachures soulignant les talus (figure 6). Les barrières sont présentes. L'impraticabilité de certains chemins pour les voitures est parfois signalée. Le genre de cultures n'est pas spécifié, mais il y a des indications de prairies marécageuses ou arborées, de bois et landes, de rares pépinières, de sapinières, de bois dérodés ou d'un pré labouré en 1806 le long de la Senne non loin de Bruxelles. Un gros trait noir sur plusieurs feuilles correspond à la limite de la forêt de Soignes, sans qu'il soit possible de connaitre la période exacte à laquelle elle se rapporte. Un grand arbre isolé, peuplier ou tilleul 
réaliste et différencié, attire l'attention, ainsi que de petites carrières de pierre blanche en quelques endroits. Un "sable rude et rouge" et une "contrée riche en Moutons" se trouvent sur les feuilles méridionales, qui portent de nombreuses références aux événements de 1815 : "le plus fort de la Bataille du 18 juin" au nord de la Haye Sainte, "position des Alliés, arrivée du quatrième corps d'armée des Prussiens, bois du Callois avec l'observatoire, la Belle Alliance, anctCabaret d'où la gde Bataille du 18 juin 1815 prit le Nom". Wautier retient donc le nom que Blücher avait repris plutôt que le choix de Wellington. Il donne le patronyme du duc britannique, prince de Waterloo, à un des cinq cabarets de Waterloo où le prince héréditaire d'Orange, blessé, avait été transporté. Plus au nord, il y a un rappel plus ancien, concernant le "vieux château du Général Jaco bâti 1698 où il est mort". Ces éléments sont d'autant plus frappants que les feuilles sont loin d'être achevées et qu'il y a même une note "à refaire" à l'intérieur du périmètre d'un bois près de Glabbais.

Figure 6. Wautier f.11 planchette 4/3 Lasne. (inachevée).

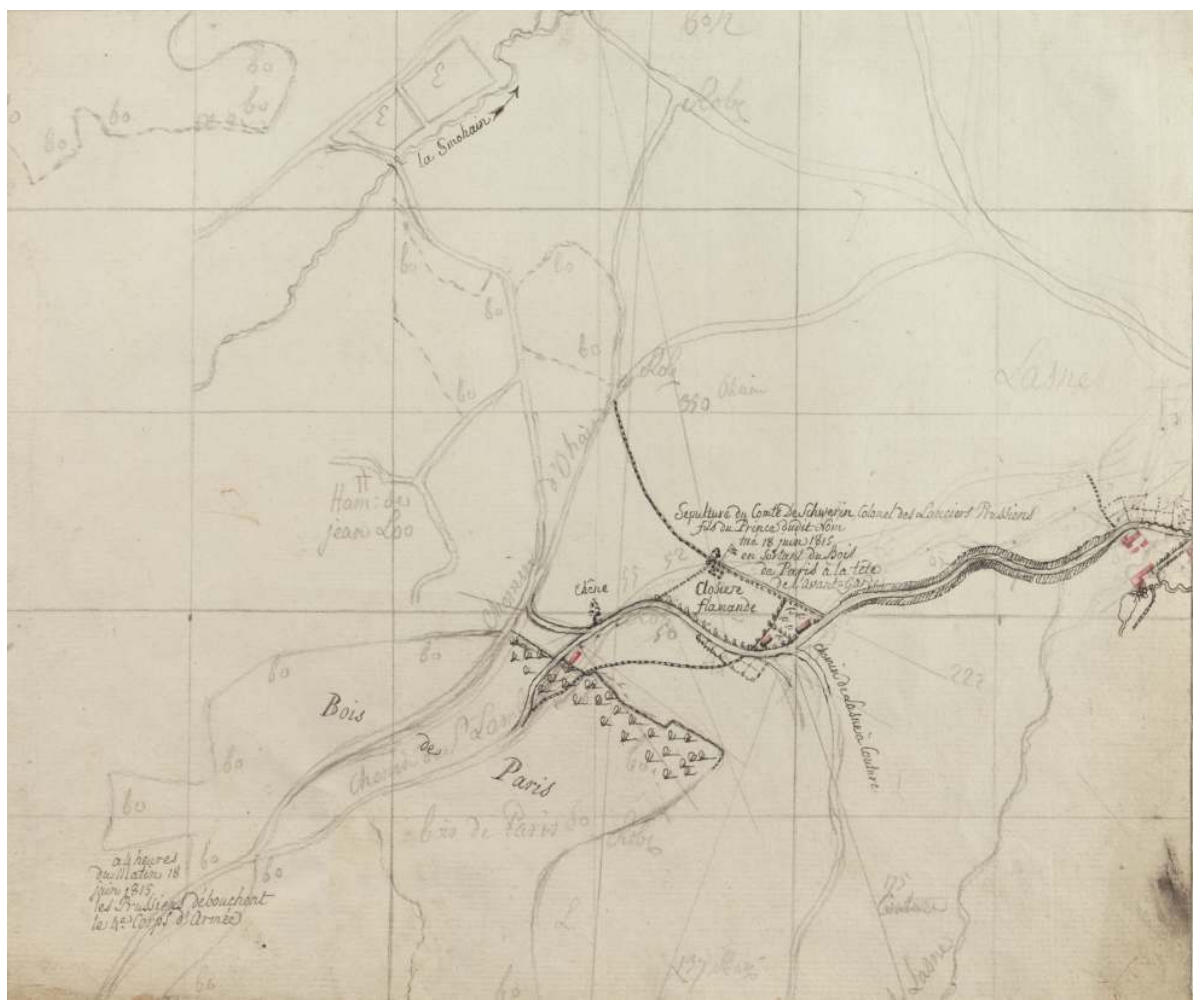

La carte se compose de douze fois quatre feuilles, chacune divisée en quatre planchettes accolées ; les renseignements originaux inscrits au verso ont été complétés, probablement par le chanoine Puissant ${ }^{14}$, ancien propriétaire du document, et par des ajouts à la Bibliothèque Royale. A l'exception des feuilles 1 et 2 , les plus occidentales, les autres portent les indications de la main de Wautier $\mathrm{Col} 50$ et $\mathrm{Col}$ 51, avec un numéro de planche et de section, souvent occultés par le rebord en toile. Ont été ajoutées au crayon bleu des lettres de A à J, subdivisées en 16, sauf $C$ en 48 (feuilles 3-4-5, 4 étant la ville de Bruxelles). Les colonnes indiquent l'agencement prévu par l'auteur, qui prolonge sa carte par de petits feuillets montrant les chaussées, à la même échelle 1/6100, donc beaucoup plus grande que celle des routes publiées comme itinéraires. Ainsi, l'esquisse pour Malines devrait venir dans la colonne 51, celle de Tubize dans la 47, les parties de la route de Mons, dans 47, 48 et 49, chaque fois avec un numéro de plan. Au verso de la chaussée de 
Mons, une "Note de ce qui doit être mesuré pour la Carte de Waterloo", douze numéros de chemins et restant de forêt, prouve que ces feuillets sont bien liés à la carte des environs de Bruxelles.

Les esquisses des routes tracées à l'encre se limitent aux assises de la voie et aux abords, encore souvent au crayon; elles reprennent les barrières et quantité d'auberges au nom évocateur comme le "ci-devant Lit tout chaud", devenu maison de garde forestier. Près de Tubize, on relève Au Soleil, à la Chaîne d'Or et à l'Aigle d'Or, établissements qui ont été retrouvés comme oeuvrant dans le même secteur sur le plan parcellaire de Popp ${ }^{15}$, édité bien plus tard (figure 7).

Une recherche semblable a été menée pour un feuillet de Malines où l'enceinte méridionale est rapidement tracée au crayon (figure 8). Différentes visées et distances sont mesurées ; l'amorce des rues et cours d'eau qui rejoignent ou traversent l'axe porte de Bruxelles-Grand-Place est reprise, l'église de Notre-Dame-au-delà-de-la-Dyle sert de point de repère et les auberges sont indiquées par leur signe et leur nom. La Grue ou De Kraan, qui existait encore en 1901 au numéro 25 de la Grote Markt, est mentionnée par Popp comme hôtel ; l'hôtel Le Miroir au 16 Koornmarkt avait ses écuries rue du Miroir ${ }^{16}$.

31 Un feuillet au nord de Malines, avec la chaussée d'Anvers, montre un moulin à tan, Schorsmolen chez Popp, à l'ouest de la route, un autre, anonyme à l'est qui est le Molen van Lier sur le plan cadastral. Le Schippershof appartenait à la fin du siècle au chanoine Scheppers de Malines et le cabaret America, près d'un ruisseau, était toujours un débit de boisson.

Ces confrontations avec la carte de Popp prouvent que le travail de Wautier n'était pas inexact et que ses méthodes, bien qu'encore mal élucidées, n'étaient certes pas méprisables. Sa carte est un compromis entre les réalisations antérieures, où beaucoup d'éléments étaient figurés en vue oblique ou cavalière, et les méthodes plus modernes qui utilisaient davantage de symboles. Ceux-ci font leur apparition chez Wautier, mais ses feuilles sont encore très "bavardes", ce qui est d'ailleurs un avantage pour qui essaie de retracer le paysage vers 1800 . Mais à quelle date précise ? Des éléments de l'ancien régime se retrouvent, tel le château de Stuyvenberg au duc de Saxe-Teschen à Laeken. La ville de Bruxelles présente un aspect égal ou antérieur à 1819 , la bataille de Waterloo est commentée, les levés de la chaussée de Ninove reportent à 1821-22. Il semblerait donc que Guillaume de Wautier se soit occupé de sa carte pendant plusieurs décennies. Les feuilles achevées sont très esthétiques et, malgré de légères inexactitudes inhérentes à ce genre de travail accompli par une seule personne, le résultat est très intéressant pour l'histoire de la cartographie et de la géographie du centre de notre pays avant l'indépendance.

\section{NOTES}

1. Catalogue de la Bibliothèque et des collections scientifiques, etc., de l'Etablissement géographique à Bruxelles... sous la direction de A.Bluff (1880), Bruxelles, VIII-91 ?pp. 
2. VOISIN A. (1836-1837), Bibliotheca Hulthemiana ou Catalogue méthodique de la riche et précieuse collection de livres et de manuscrits délaissés par Ch.Van Hulthem, Gand, 1836-1837, 6 vol. (37685 numéros répertoriant les imprimés et 1016 les manuscrits pour un total de quelque 64000 unités). 3. Charles Van Hulthem (Gand, 1764-1832). Formation de juriste; suivit également des cours de science et de bibliothéconomie. Mêlé à la politique et à la direction des arts. Très tôt, Van Hulthem commença une collection de livres et d'objets d'art, en provenance e.a. des couvents supprimés. Mécène, conservateur de la bibliothèque de la ville de Bruxelles, il est surtout connu comme grand amateur de plantes. Il fut à la base de l'amélioration de l'horticulture et introduisit de nombreuses espèces en Belgique. Secrétaire perpétuel de l'Académie en 1817. Sa collection de livres et de manuscrits couvrait presque tous les domaines des connaissances. Voir les études dans le catalogue de l'exposition :

Karel van Hulthem 1764-1832. Tentoonstelling... (1964), Brussel, Albert I-Bibliotheek, Gent, Universiteitsbibliotheek, Brussel, Koninklijke Bibliotheek, 331 pp. , ill. (existe aussi en français).

4. BATCHELOR C. (2005), "The mythical mountains of Kong", in BIMCC Newsletter, Nr 23, pp. 19-21.

5. Karel Van Hulthem, o.c., pp. 72-73, 91-92 et passim.

6. Jean-Baptiste De Bouge (1757-1833), cartographe formé à l'Académie militaire de Malines, dessinateur chez Ferraris, graveur. Publia dès 1786 une carte des Pays-Bas autrichiens, réduction de la carte marchande de Ferraris; plusieurs éditions retouchées sous différents titres jusqu'en 1815. Employé à la Chambre des Comptes, engagé par le roi de Prusse, puis au Dépôt de la Guerre de Hollande, De Bouge publia de nombreux travaux personnels. Citons sa grande carte de l'Europe, la carte de l'Europe centrale, des Tenances de poste, de la Révolution brabançonne, du royaume des Pays-Bas en 1823 ; plusieurs Théâtres de la guerre selon les événements en Europe, des guides des voyageurs, des plans de Bruxelles, etc., repris sous 106 numéros dans l'Annexe 1 et passim dans :

7. Comme rien de précis n'est actuellement connu pour Madame B. ?Monborne, il est impossible de dire si elle est réellement l'auteur des cartes, du moins partiellement, comme le ferait croire sa signature dans la marge ou l'intitulé de la rubrique 1835 de la Revue bibliographique de 1824 . Dans un tout autre domaine, une dame B. ?Monborne est l'auteur de trois livres parus à Paris en 1834 et 1835, conservés à la Bibliothèque Nationale. Il s'agit de : La veste de satin ou les Trois Règnes, 1789 - 1798 - 1811; Une victime, esquisse littéraire; Deux originaux (roman à caractère sociosentimental). Etienne Wauty nous a signalé que des Monborne étaient originaires de GrandeBretagne et étaient établis aux environs de Dunkerque.

8. LEMOINE-ISABEAU C., o.c., pp. 153-154.

9. HENS-VERCAUTEREN L. (1973), "De topografische kaarten van de Zuidelijke Nederlanden, 1815-1830", in Tijdschrift van de Belgische Vereniging voor Aardrijkskundige Studies, XLII, 2, pp. 333-455, ill. - JANSSENS, F. (1998), De kartering in België tijdens het Hollands Bewind, 1815-1830 : de werking van de afdeling Militaire Verkenningen en een nauwkeurigheidsanalyse van haar kaartcollectie, Leuven, K.U.L., doctoraatsverhandeling, promotor prof. Frans Depuydt.

10. Un détail de la feuille 29 pour la région correspondante a été reproduit en couleurs à l'échelle, in DUVOSQUEL, J.M. \& LEMOINE-ISABEAU, C. (1980), La région de Comines-Warneton. Sept siècles de documents cartographiques et iconographiques, Bruxelles, pp. 67-68, fig.48.

11. Guillaume de Wautier de Beren (1757-après 1825). Cartographe formé à l'Artillerie et à l'Ecole d'Hydraulique ; a participé à la Carte des Limites de la Flandre et de la France. Wautier travailla aux fortifications de Bruxelles; attaché à la Chambre des Comptes (collègue de De Bouge). Sa grande carte de Bruxelles, qu'il dessina vraisemblablement pendant des décennies, est restée manuscrite. En 1810, il publia une Carte topographique des environs de Bruxelles avec un guide. Voir aussi : LEMOINE-ISABEAU C., o.c., pp. 27, 93-94, 295 et passim.

12. DANCKAERT L. (1968), L'évolution territoriale de Bruxelles. La cartographie de +/- 1550 à 1840, Bruxelles, 164 pp. , ill. (Pour Wautier, voir $n^{\circ}$ 77, pp. 93-94 ; Monborne, $n^{\circ}$ 87, pp. 104-105). - ID. 
(1989), Bruxelles. Cinq siècles de cartographie, Tielt, 144 pp., ill. (Wautier $n^{\circ} 73$, pp. 99, 104-105 ; Monborne ${ }^{\circ}$ 74, pp. 103, 105-106).

13. ID. (1989), "Guillaume De Wautier", in RAMAN A. \& COCKSHAW P., Bibliothèque royale de Belgique. Cent cinquantenaire de l'ouverture au public, pp. 165-167, ill.

14. Par son étude des manuscrits des abbayes, le chanoine Puissant (Renaix 1860 - Mons 1934) acquit une grande connaissance de l'histoire du Hainaut. Son intérêt pour l'archéologie l'amena à pratiquer des fouilles et à restaurer plusieurs édifices anciens, tels les châteaux de Havré et d'Ecaussinnes-Lalaing, ainsi que des retables. Il était membre de nombreuses sociétés scientifiques. Voir : CHAMPAGNE P. (1965-66), "Puissant (Edmond Félix)", in Bibliographie nationale , XXXIII, col. 604-608.

15. L'utilité des plans cadastraux de P.C.Popp est renforcée par l'existence des matrices qui répertorient le nom et la profession du propriétaire des parcelles, parfois de l'exploitant. La date n'est malheureusement pas indiquée, mais la situation des communes du Brabant remonte vraisemblablement à 1855-65. La province d'Anvers a été publiée partiellement après le décès de Popp en 1879.

16. VAN CASTER W. (1901/1975), Namen der straten van Mechelen en korte beschrijving hunner... oude gebouwen, Brussel, Kultuur en Beschaving, VII-335 pp. (Anastatische herdruk van de uitgave van 1901).

\section{RÉSUMÉS}

Avant l'Indépendance, des ouvrages de géographie ou de cartographie, souvent à caractère didactique, étaient disponibles en Belgique, même s'ils n'étaient pas nécessairement d'usage général. Le bibliophile Charles Van Hulthem en possédait un assez grand nombre. L'Atlas Universel de Philippe Vandermaelen avait rencontré un franc succès. Une série de cartes éditées par Madame Monborne ne semble, par contre, pas avoir été largement diffusée. Pour la région frontalière du sud-ouest, les feuilles montrent des similitudes troublantes avec les cartes des Reconnaissances militaires ou Militaire Verkenningen, restées manuscrites.

Les feuilles des environs de Bruxelles de Monborne ont permis une comparaison avec la carte manuscrite très détaillée de Guillaume de Wautier, presque contemporaine, qui présente un contenu assez proche, malgré un aspect fort différent. Cette carte à grande échelle comporte des feuilles achevées et d'autres en cours d'élaboration, parfois même à peine esquissées.

Vóór de Onafhankelijkheid waren boeken over aardrijkskunde of cartografie, dikwijls met een didactisch karakter, beschikbaar in België, zij niet algemeen verspreid. Bibliofiel Karel Van Hulthem bezat er een vrij groot aantal van. Philippe Vandermaelens Atlas Universel kende een groot succes. Een reeks door Mevrouw Monborne uitgegeven kaarten was echter veel minder bekend. De bladen die een strook bij de zuidwestelijke grens weergeven tonen een opvallende gelijkenis met de handgetekende kaarten van de Militaire Verkenningen.

De bladen van het Brusselse van Monborne werden vergeleken met de zeer gedetailleerde, bijna gelijktijdig opgemaakte manuscriptkaart van Guillaume de Wautier. Verwant qua inhoud maar zeer verschillend van aspect bestaat deze grootschalige kaart uit afgewerkte, in opmaak zijnde of zelfs amper geschetste bladen. 
INDEX

Mots-clés : collection géographique en Belgique, Van Hulthem, cartographie belge au début du XIXe s., Monborne, Wautier

Trefwoorden Belgische aardrijkskundige verzameling, Van Hulthem, Belgische cartografie begin 19e e., Monborne, Wautier

\section{AUTEUR}

\section{LISETTE DANCKAERT}

Chef de section honoraire à la Bibliothèque royale de Belgique 